\title{
Automatic Derivation of Multiharmonic Formulations for Nonlinear Electromechanical Problems with Time Dependent Mesh Deformation
}

\author{
Alexandre Halbach and Christophe Geuzaine \\ University of Liège, Department of Electrical Engineering and Computer Science, \\ Montefiore Institute B28, B-4000 Liège, Belgium \\ \{alexandre.halbach, cgeuzaine\}@ulg.ac.be
}

\begin{abstract}
This paper describes a method to automatically derive multiharmonic finite element formulations for coupled, nonlinear electromechanical problems. It focuses on models of electrically actuated micromembranes using both a staggered and a monolithic Newton iteration scheme. Two- and three-dimensional examples highlight the main properties of the proposed method.
\end{abstract}

\section{Introduction}

Many applications require the computation of the steady-state response to a time-harmonic excitation. In order to evaluate the cross-talk between electrically actuated vibrating micromembranes for example, one may excite a given membrane in the array using a harmonic electric potential and compute the perturbation induced on the other membranes in the array. To solve such coupled electromechanical problems [1] one can use a time-stepping method (e.g. Newmark's method [2], [3] in combination with a Newton linearisation at each step) and wait until the steady-state is reached. As an alternative, for linear problems it is straightforward to perform a harmonic resolution (in Fourier space) to obtain the desired steadystate solution. For nonlinear problems however additional harmonics appear. Truncating the Fourier series of the unknown fields and approximating the Fourier coefficients by finite elements in this case leads to a large coupled nonlinear system. This method is called the multiharmonic or harmonic balance finite element method. It has been shown in [4] that the convergence of the Fourier approximation is generally of order $N^{-1}$ (where $N$ is the number of Fourier terms considered in the truncation) but can be much faster for simple harmonic excitations [5] [6].

The multiharmonic method has already been investigated in several fields [5], [7], [8], [9], [10], [11]. Its effective use on large scale applications is however impeded by two main factors. On the one hand the size of the nonlinear system is multiplied by $N$ compared to the time-domain approach. On the other hand the derivation of the equation terms in the multiharmonic formulation (even for $N=2$ ) can become extremely tedious when done manually.

In this paper we propose a general framework to derive the terms of the multiharmonic formulations automatically based on symbolic computation. This allows to derive systematically, without user effort, the multiharmonic formulations of (multi-) harmonically excited nonlinear systems, even when the time dependent unknown fields appear in nonpolynomial form. As an application example, we apply this framework to the analysis of electrically actuated micromembrane arrays, discretised using high order finite elements. The multiharmonically vibrating mesh is naturally handled in the nonlinear system, which is either solved in a staggered iterative scheme or using a monolithic Newton iteration. For conciseness, the handling of the large scale algebraic systems resulting from the multiharmonic formulation will not be addressed in this paper. As was recently shown in [12], domain decomposition approaches can be advantageously used in this regard.

The paper is organised as follows. In section 2 the multiharmonic resolution framework is presented on a simple 1D example before being applied to the full nonlinear electromechanical problem in section 3. Section 4 presents numerical results on 2D and 3D models of electrically actuated micromembrane arrays.

\section{Multiharmonic Formulation}

\section{A. Electrostatic Formulation on the Undeformed Mesh}

Let us consider a 1D electrostatic problem solved in terms of the electrostatic potential $v$ on a multiharmonically vibrating mesh, deformed by the mechanical displacement $u$. The system is excited via a timeharmonic Dirichlet boundary condition on the electric potential $\bar{v}=V_{1} \sin \left(2 \pi f_{0} t\right)$ on an electrode. A reference potential of 0 is imposed on the ground. Let us assume that the electric potential solution $v$ and the mechanical displacement $u$ can be approximated by the truncated Fourier series $v=V_{1} \sin \left(2 \pi f_{0} t\right)+V_{3} \sin \left(3 \cdot 2 \pi f_{0} t\right)$ and $u=U_{0}+U_{2} \cos \left(2 \cdot 2 \pi f_{0} t\right)$, i.e. considering the first and third harmonic for the electric potential and the constant term and second harmonic for the displacement. While this limited expansion is chosen for the simplicity of the following analytic calculations, it will be seen in Section 4 that it already leads in practice to very good numerical results. The goal of the multiharmonic resolution is to find the Fourier coefficients $V_{1}, V_{3}, U_{0}$ and $U_{2}$.

Since the mesh deformation $u$ is decomposed as a sum of harmonics, integration on the mesh deformed by $u$ must be handled carefully. All the quantities are brought back to the undeformed mesh [13], by introducing the change of 
variables for the 1D $x$ coordinate $x^{*}=x+u_{x}$ with Jacobian $J$. Denoting by $\Omega$ the undeformed configuration and by $\Omega^{*}$ the deformed one and using relations $d \Omega^{*}=|J| d \Omega$ and $\nabla^{*}=J^{-1} \nabla$ leads to the following weak formulation of the electrostatic problem: Find $v$ in an appropriate function space such that

$$
-\int_{\Omega^{*}} \varepsilon\left(\nabla^{*} v\right)^{T} \nabla^{*} v^{\prime} d \Omega^{*}=0
$$

holds for all test functions $v^{\prime}$ with $v=\bar{v}$ on the electrode and 0 on the ground, and where $\varepsilon$ is the electric permittivity. On the undeformed mesh the formulation becomes

$$
-\int_{\Omega} \varepsilon(\nabla v)^{T} J^{-T} J^{-1} \nabla v^{\prime}|J| d \Omega
$$

with

$$
J=\frac{\partial x^{*}}{\partial x}=\left[1+\frac{\partial u_{x}}{\partial x}\right], \quad J^{-1}=\frac{\partial x}{\partial x^{*}}=\frac{1}{|J|} \cdot[1]
$$

and $|J|=1+\frac{\partial u_{x}}{\partial x}$ in this one dimensional setting. Equation (2) can be rewritten as:

$$
-\int_{\Omega} \varepsilon \frac{\partial v}{\partial x} \frac{\partial v^{\prime}}{\partial x} \frac{1}{|J|} d \Omega=0
$$

It should be noted that in $2 \mathrm{D}$ and $3 \mathrm{D}$ extra Jacobian terms appear. Some of those can be neglected in small displacement applications but not in the kind of vibrating micromembrane test cases we consider, where the displacements can be large compared to the overall geometrical dimension of the problem. Also note that because of the abrupt change of $J$ and $|J|$ between a solid material and a non-solid material like e.g. air, one should avoid any kind of averaging for the Jacobian at these interfaces.

\section{B. Multiharmonic Expansion}

In order to obtain the final multiharmonic formulation, the non-polynomial factor $G:=\frac{1}{|J|}$ is first computed in weak form: Find $G$ such that

$$
\int_{\Omega_{e}} G|J| G^{\prime} d \Omega=\int_{\Omega_{e}} G^{\prime} d \Omega
$$

holds for appropriate test functions $G^{\prime} . G$ itself is computed using a multiharmonic resolution. In practice it is well approximated with two Fourier coefficients: $G=$ $G_{0}+G_{2} \cos \left(2 \cdot 2 \pi f_{0} t\right)$.

The next step could then simply be to symbolically replace $v$ and $\frac{1}{|J|}$ (and $u$ in $2 \mathrm{D}$ and $3 \mathrm{D}$ ) by their truncated Fourier expansion and then expand the whole formulation and multiply the sines and cosines together using recursively the following four identities to leave only sines and cosines of degree one but at higher frequencies:

$$
\begin{array}{ll}
\cos (a) \cdot \cos (b) & =\frac{\cos (a+b)}{2}+\frac{\cos (a-b)}{2} \\
\sin (a) \cdot \sin (b) & =\frac{\cos (a-b)}{2}-\frac{\cos (a+b)}{2} \\
\sin (a) \cdot \cos (b) & =\frac{\sin (a+b)}{2}+\frac{\sin (a-b)}{2} \\
\cos (a) \cdot \sin (b) & =\frac{\sin (a+b)}{2}-\frac{\sin (a-b)}{2}
\end{array}
$$

Doing so at this step would however make the number of expanded symbolic terms increase dramatically. In 1D it can be shown that it increases as $N^{2}$ because of the product between $G$ and $\frac{\partial v}{\partial x}$, while in 2D it increases as $N^{4}$. In order to avoid this issue and limit the explosion of the number of terms one can multiharmonically precompute all products of terms that are known, i.e. all terms but the unknown and the test function. Best is to multiply all known terms 2 by 2 recursively until the whole term has been computed.

In the $1 \mathrm{D}$ case there is only the $\frac{1}{|J|}$ known term so that there is no need to perform the previous step. One can immediately move to the next steps:

- replace the multiplied known term and the unknown by their truncated Fourier series;

- expand the formulation;

- apply time derivatives to the sines and cosines;

- use (5) to transform the sines and cosines powers and products into sums of higher frequency sines and cosines.

This amounts to transforming (3) into:

$$
\begin{aligned}
& -\int_{\Omega} \varepsilon \frac{\partial v}{\partial x} \frac{\partial v^{\prime}}{\partial x} \frac{1}{|J|} d \Omega=0 \\
\Leftrightarrow & -\int_{\Omega} \varepsilon \frac{\partial\left(V_{1} \sin \left(2 \pi f_{0} t\right)+V_{3} \sin \left(3 \cdot 2 \pi f_{0} t\right)\right)}{\partial x} \frac{\partial v^{\prime}}{\partial x} \\
& \cdot\left(G_{0}+G_{2} \cos \left(2 \cdot 2 \pi f_{0} t\right)\right) d \Omega=0 \\
\Leftrightarrow & -\int_{\Omega} \varepsilon\left[G_{0} \frac{\partial V_{1}}{\partial x} \sin \left(2 \pi f_{0} t\right)+G_{0} \frac{\partial V_{3}}{\partial x} \sin \left(3 \cdot 2 \pi f_{0} t\right)\right. \\
& +G_{2} \cos \left(2 \cdot 2 \pi f_{0} t\right) \frac{\partial V_{1}}{\partial x} \sin \left(2 \pi f_{0} t\right) \\
& \left.+G_{2} \cos \left(2 \cdot 2 \pi f_{0} t\right) \frac{\partial V_{3}}{\partial x} \sin \left(3 \cdot 2 \pi f_{0} t\right)\right] \frac{\partial v^{\prime}}{\partial x} d \Omega=0 \\
\Leftrightarrow & -\int_{\Omega} \varepsilon\left[\left(G_{0} \frac{\partial V_{1}}{\partial x}-\frac{1}{2} G_{2} \frac{\partial V_{1}}{\partial x}+\frac{1}{2} G_{2} \frac{\partial V_{3}}{\partial x}\right)\right. \\
& \cdot \sin \left(2 \pi f_{0} t\right)+\left(G_{0} \frac{\partial V_{3}}{\partial x}+\frac{1}{2} G_{2} \frac{\partial V_{1}}{\partial x}\right) \sin \left(3 \cdot 2 \pi f_{0} t\right) \\
& \left.+\left(\frac{1}{2} G_{2} \frac{\partial V_{3}}{\partial x}\right) \sin \left(5 \cdot 2 \pi f_{0} t\right)\right] \frac{\partial v^{\prime}}{\partial x} d \Omega=0
\end{aligned}
$$

which is valid for any time $t$ and can thus be split into three independent equations, with the sine terms removed. Taking the equations corresponding to the Fourier expansion of $v$, i.e. the terms multipled by $\sin \left(2 \pi f_{0} t\right)$ and the ones multiplied by $\sin \left(3 \cdot 2 \pi f_{0} t\right)$ gives an excellent approximation of the actual electrostatic formulation and 
leads to the final multiharmonic formulation: Find $V_{1}$ and $V_{3}$ such that

$$
\left\{\begin{array}{l}
\int_{\Omega} \varepsilon\left(G_{0} \frac{\partial V_{1}}{\partial x}-\frac{1}{2} G_{2} \frac{\partial V_{1}}{\partial x}+\frac{1}{2} G_{2} \frac{\partial V_{3}}{\partial x}\right) \frac{\partial v^{\prime}}{\partial x} d \Omega=0 \\
\int_{\Omega} \varepsilon\left(G_{0} \frac{\partial V_{3}}{\partial x}+\frac{1}{2} G_{2} \frac{\partial V_{1}}{\partial x}\right) \frac{\partial v^{\prime}}{\partial x} d \Omega=0
\end{array}\right.
$$

holds for appropriate test functions $v^{\prime}$.

This system can be rewritten in matrix form:

$$
\left[\begin{array}{ll}
K_{V_{1} V_{1}} & K_{V_{1} V_{3}} \\
K_{V_{3} V_{1}} & K_{V_{3} V_{3}}
\end{array}\right]\left[\begin{array}{l}
V_{1} \\
V_{3}
\end{array}\right]=\left[\begin{array}{l}
0 \\
0
\end{array}\right]
$$

where each of the four blocks can be generated using a usual monoharmonic finite element assembler.

As can be seen, even for linear electrostatic problems the harmonics $V_{1}$ and $V_{3}$ can be coupled if the mesh is deformed. To understand that this makes sense simply consider a mechanical membrane vibrating harmonically as a sine wave and a constant applied electrostatic voltage between two electrodes. Even though the electrostatic voltage on the electrode is constant the voltage inside the membrane will vary with time and thus the overall voltage will have a constant component plus a harmonic component. In case the membrane displacement is a constant or simply zero then the electric potential harmonics are uncoupled and the off-diagonal blocks $K_{V_{1} V_{3}}$ and $K_{V_{3} V_{1}}$ are zero as can be seen in (7) when $G_{2}$ is set to zero.

\section{Implementation Aspects}

It should be no surprise that the multiharmonic formulation for the $2 \mathrm{D}$ and in particular the $3 \mathrm{D}$ case will contain a lot of terms, since the computation on the vibrating mesh is brought back via a coordinate change on the undeformed mesh. Nevertheless, all that is needed for the symbolic calculations is an algorithm able to perform symbolic expansions of polynomials into sums of monomials (e.g. $2(a+b)^{2}=2 a^{2}+2 a b+2 b^{2}$ ), readily available as expand in the Matlab software for example, and a function able to transform powered sines, cosines and products thereof into sums of non powered higher frequency harmonics (e.g. replace $\cos (x)^{2}$ by $\frac{1}{2}+\frac{1}{2} \cos (2 x)$ ) using recursively the four trigonometric identities in (5).

In practice the symbolic computation is a matter of seconds for the assembly of the stiffness matrix of the electrostatic problem while the evaluation of the Jacobian matrix for Newton's iteration is significantly more expensive (up to 2 minutes with 4 harmonics on the 3D test case presented in section 4 in our non-optimised Matlab code).

\section{Nonlinear Electromechanical Coupling}

We can readily use the above multiharmonic framework to compute the steady-state behavior of 2D and 3D electromechanical systems. In order to avoid lengthy derivations only the $2 \mathrm{D}$ formulation is presented in what

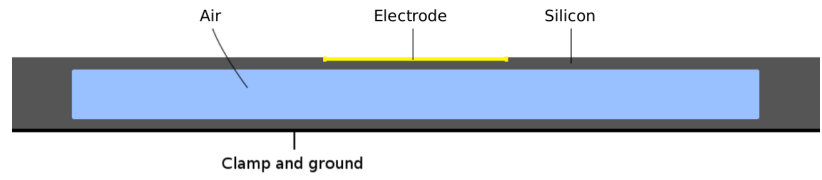

Fig. 1. One membrane of the 2 by $12 \mathrm{D}$ micromembrane array.

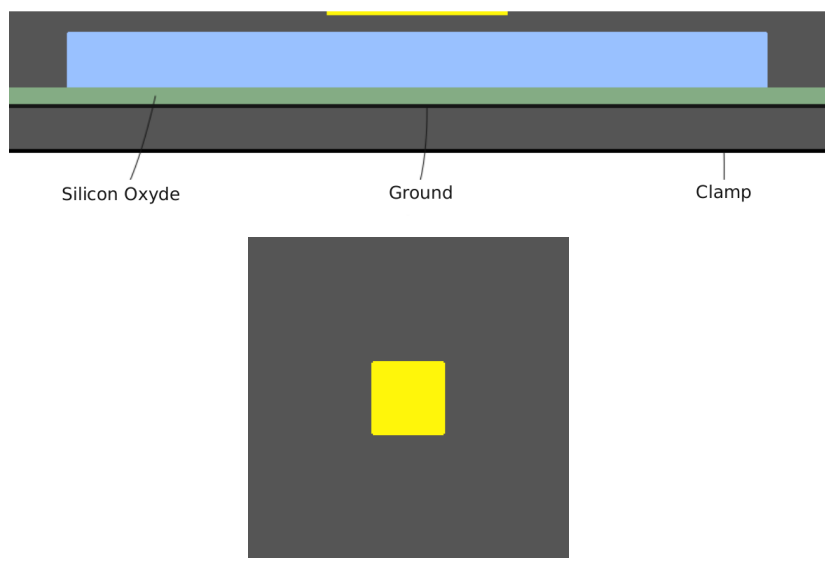

Fig. 2. One membrane of the 2 by 2 3D micromembrane array - Side and top view.

follows, in its time dependent form. This form is what the multiharmonic method is fed with so as to automatically derive the corresponding multiharmonic formulation for a required number of harmonics.

Consider an electromechanical system with a mechanical subdomain $\Omega_{m}$ and an electric subdomain $\Omega_{e}$. (A star superscript will here again denote a deformation by the displacement field.). Let $v$ be the electrostatic potential defined on $\Omega=\Omega_{e}, u$ the displacement field defined on $\Omega_{m} \subset \Omega$ with components $u_{x}$ and $u_{y}$ and $\mathcal{M}$ the $2 \mathrm{D}$ elasticity operator defined by $\mathcal{M}(u)=\left[\begin{array}{lll}\frac{\partial u_{x}}{\partial x} & \frac{\partial u_{y}}{\partial y} & \frac{\partial u_{x}}{\partial y}+\frac{\partial u_{y}}{\partial x}\end{array}\right]^{T}$. We consider the following weak formulation of the electrostatic problem with Dirichlet boundary conditions on $\Gamma_{e} \subset \partial \Omega_{e}$ : Find $v$ such that

$$
-\int_{\Omega_{e}^{*}} \varepsilon(\nabla v)^{T} \nabla v^{\prime} d \Omega^{*}=0, v=\bar{v} \text { on } \Gamma_{e},
$$

holds for appropriate test functions $v^{\prime}$. Defining $E_{x}=\frac{\partial v}{\partial x}$ and $E_{y}=\frac{\partial v}{\partial y}$ and using the Frobenius matrix product $A: B=\sum_{i, j} A_{i, j} B_{i, j}$, we consider the following weak formulation of the $2 \mathrm{D}$ linear elasticity problem with Dirichlet 
boundary conditions on $\Gamma_{m} \subset \partial \Omega_{m}$ : Find $u$ such that

$$
\begin{gathered}
\int_{\Omega_{m}} \frac{E}{1-v^{2}}\left[\begin{array}{ccc}
1 & v & 0 \\
v & 1 & 0 \\
0 & 0 & \frac{1-v}{2}
\end{array}\right] \mathcal{M}(u)^{T} \mathcal{M}\left(u^{\prime}\right) d \Omega \\
+\int_{\Omega^{*}} \frac{\varepsilon}{2}\left[\begin{array}{cc}
E_{x}^{2}-E_{y}^{2} & 2 E_{x} E_{y} \\
2 E_{x} E_{y} & E_{y}^{2}-E_{x}^{2}
\end{array}\right]:\left[\begin{array}{cc}
\nabla u_{x}^{\prime} & \nabla u_{y}^{\prime}
\end{array}\right] d \Omega^{*} \\
-\int_{\Omega_{m}} \rho \frac{\partial^{2} u^{T}}{\partial t^{2}} u^{\prime} d \Omega=0, \quad u=\bar{u} \text { on } \Gamma_{m},
\end{gathered}
$$

holds for appropriate test functions $u^{\prime}$, where $v$ is Poisson's ratio, $E$ Young's modulus and $\rho$ the mass density (all homogeneous anisotropic). The second term in 10 is the (nonlinear in $v$ ) electrostatic force computed using the virtual work principle, see e.g. [14].

We consider two solution schemes for the coupled electromechanical problem. The first is a staggered coupling that consists in solving the electrostatic and elasticity formulations (9)-(10) in alternance. In this case only the assembly of the stiffness matrix of the electrostatic formulation (9) requires the multiharmonic framework. Indeed, all the harmonics in the elasticity formulation are uncoupled and can be solved independently. The second solution scheme consists in solving (9)-(10) at once in a monolithic manner, using a Newton-Raphson scheme. In this case, the linearized coupling terms have to be computed to obtain the Jacobian matrix [15] of the coupled system. This matrix involves higher order powers of the unknown fields leading to a massive amount of terms in the multiharmonic expansion.

\section{Numerical Results}

We consider both a 2D model of a $2 \times 1$ vibrating micromembrane array (Fig. 1, not to scale) and a more complex 2x2 3D model (Fig. 2, also not to scale). One membrane in the $2 \mathrm{D}$ array consists of a silicon membrane vibrating in the air due to an electrostatic force coming from a sine voltage applied between the electrode and the ground. In the 3D case the electric ground is separated from the mechanical clamp by an insulating silicon oxide layer and a silicon bulk. Formulation (10) is solved in the silicon and silicon oxyde while formulation (9) is solved in the silicon, silicon oxyde and in the air. The mesh is made up of 3546 second order quadrangles in 2D and 1024 second order hexahedra in 3D. Homogeneous Dirichlet boundary conditions are applied on the clamp for the elasticity formulation and on the ground for the electrostatic formulation.

For the numerical tests, the following geometrical and material characteristics have been used: membrane length of $50 \mu \mathrm{m}$, thin support pillars thickness of about $5 \mu \mathrm{m}$, membrane thickness $0.2 \mu \mathrm{m}$, height of about $5 \mu \mathrm{m}$, electrode length of $10 \mu \mathrm{m}$; silicon and air with electric permittivity $\varepsilon_{\text {silicon }}=3.9 \cdot 8.854 \cdot 10^{-12} \mathrm{~F} / \mathrm{m}$ and $\varepsilon_{\text {air }}=$ $8.854 \cdot 10^{-12} \mathrm{~F} / \mathrm{m}$, Young's modulus $E=150 \cdot 10^{9} \mathrm{~N} / \mathrm{m}^{2}$
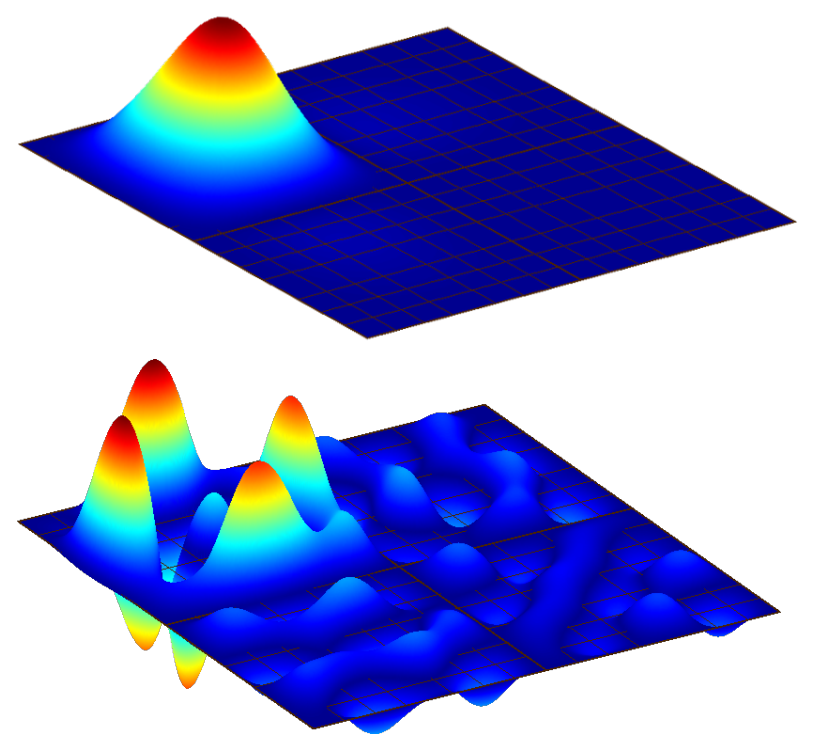

Fig. 3. Skin of the first and second harmonic displacement (not to scale) in a 3D 2 by 2 micromembrane array excited by a sine electrode to ground voltage on the upper left cell - flipped for illustration.

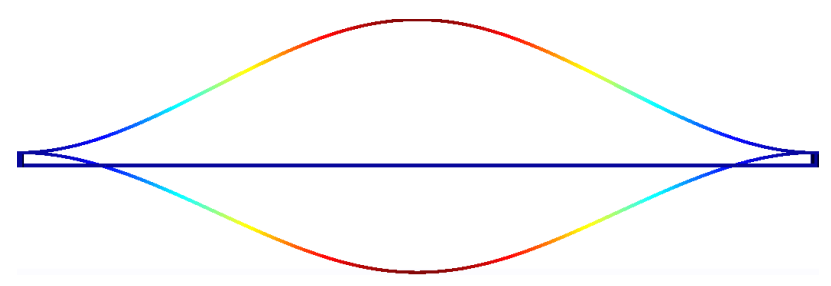

Fig. 4. First and second displacement harmonic (both exagerated by a factor 1000) on the only excited membrane of the 2D $2 \times 1$ micromembrane array when the excitation potential is $5 \mathrm{~V}$ at $100 \mathrm{kHz}$.

and Poisson's ratio $v=0.3$. The $5 \mathrm{~V}, 100 \mathrm{kHz}$ electrodeto-ground excitation voltage is applied on a single of the 2 or 4 membranes. With a sine voltage excitation it has been observed that the electric potential solution $v$ and mechanical displacement $u$ can be expanded as: $v=V_{1} \sin \left(2 \pi f_{0} t\right)+V_{3} \sin \left(3 \cdot 2 \pi f_{0} t\right)+V_{5} \sin \left(5 \cdot 2 \pi f_{0} t\right)+\ldots$ and $u=U_{0}+U_{2} \cos \left(2 \cdot 2 \pi f_{0} t\right)+U_{4} \cos \left(4 \cdot 2 \pi f_{0} t\right)+\ldots$ Note that if an extra continuous bias was added to the excitation, the extra Fourier coefficient in the excitation would be automatically handled exactly in the same way except

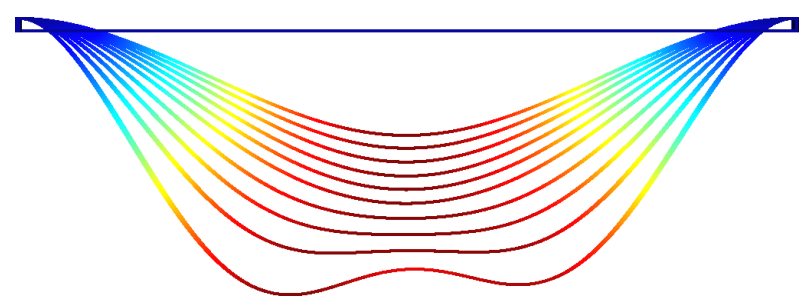

Fig. 5. First ten displacement harmonics (not to scale and flipped) on the only excited membrane of the 2D 2x1 micromembrane array when the excitation potential is $5 \mathrm{~V}$ at $100 \mathrm{kHz}$. For clarity the harmonic numbers have been ordered ascendingly from the north to the south of the figure. 


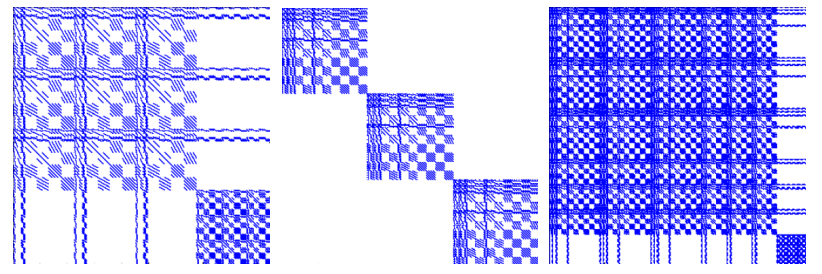

Fig. 6. From left to right: sparsity pattern of the electrostatic stiffness matrix, elasticity stiffness matrix and electromechanic Jacobian matrix for three harmonics per unknown field (2D with a third order quadrilateral mesh).

that there would be twice as many harmonics to consider in the Fourier expansion: $v=V_{0}+V_{1} \sin \left(2 \pi f_{0} t\right)+V_{2} \cos (2$. $\left.2 \pi f_{0} t\right)+V_{3} \sin \left(3 \cdot 2 \pi f_{0} t\right)+V_{4} \cos \left(4 \cdot 2 \pi f_{0} t\right)+V_{5} \sin (5$ $\left.2 \pi f_{0} t\right)+\ldots$ and $u=U_{0}+U_{1} \sin \left(2 \pi f_{0} t\right)+U_{2} \cos (2$. $\left.2 \pi f_{0} t\right)+U_{3} \sin \left(3 \cdot 2 \pi f_{0} t\right)+U_{4} \cos \left(4 \cdot 2 \pi f_{0} t\right)+U_{5} \sin (5$. $\left.2 \pi f_{0} t\right)+\ldots$

Fig. 3 illustrates the 2 by 2 3D array beyond the first resonance mode. The two harmonics of the mechanical displacement are depicted: the first Fourier coefficient $U_{0}$ has a low wavenumber while the second one, $U_{2}$, has a higher wavenumber. Fig. 4 illustrates the two first harmonics of the mechanical displacement in the 2 by $12 \mathrm{D}$ array below the first resonance mode, when the excitation voltage is $5 \mathrm{~V}$ and its frequency $100 \mathrm{kHz}$. The harmonic on the bottom is the constant harmonic while the one on the top vibrates around the bottom one at a frequency of $200 \mathrm{kHz}$. From a physical point of view this makes sense. For a very low excitation voltage and frequency one expects to get same absolute values for the amplitudes of both harmonics. Fig. 5 illustrates the higher harmonics (from the first to the tenth) on the same problem. Amplitudes are not to scale and some have been flipped for illustration.

Fig. 6 shows the sparsity pattern of the finite element matrix for the multiharmonic electrostatic formulation on the left, for the elasticity in the middle and for the electromechanical Jacobian on the right when three terms are considered in the Fourier series of both the displacement field $u$ and the electric potential field $v$. For the electrostatic and Jacobian matrices one can see two main parts: the part corresponding to the solid region in the bottom right corner and the remaining region on the top left corner. The Jacobian matrix is made up of both the three electric potential harmonics and the three displacement harmonics. As expected the electrostatic and Jacobian matrices have their harmonics coupled. They are not however for the elasticity matrix, since the elasticity formulation is linear and must be computed on the undeformed mesh. This matrix need not be generated using the multiharmonic method. It can be generated in a classical way and LU decomposed for every harmonic independently.

The solution of both the staggered and the monolithic multiharmonic formulations has been validated against

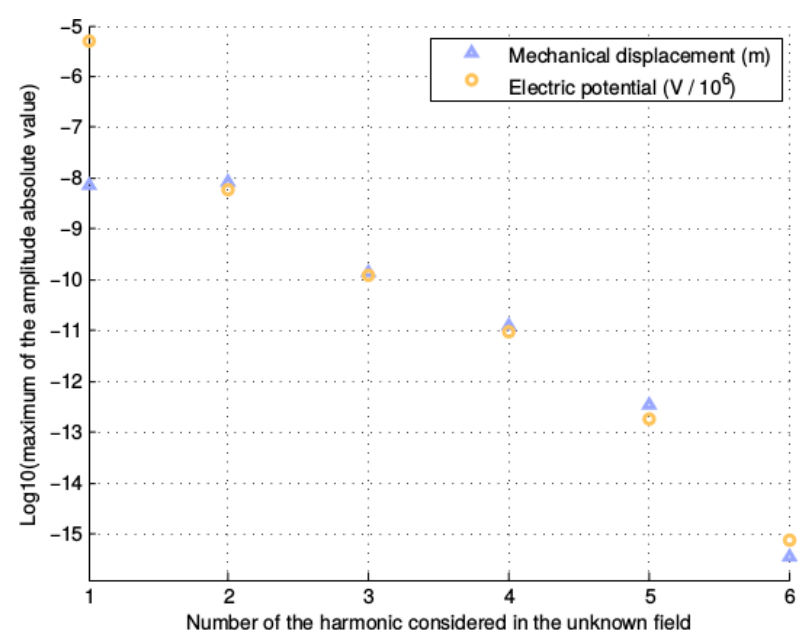

Fig. 7. Maximum absolute value displacement $(\mathrm{m})$ and electric potential $\left(\mathrm{V} / 10^{6}\right)$ for every of the six first harmonics when the excitation potential is $5 \mathrm{~V}$ at $100 \mathrm{kHz}$.

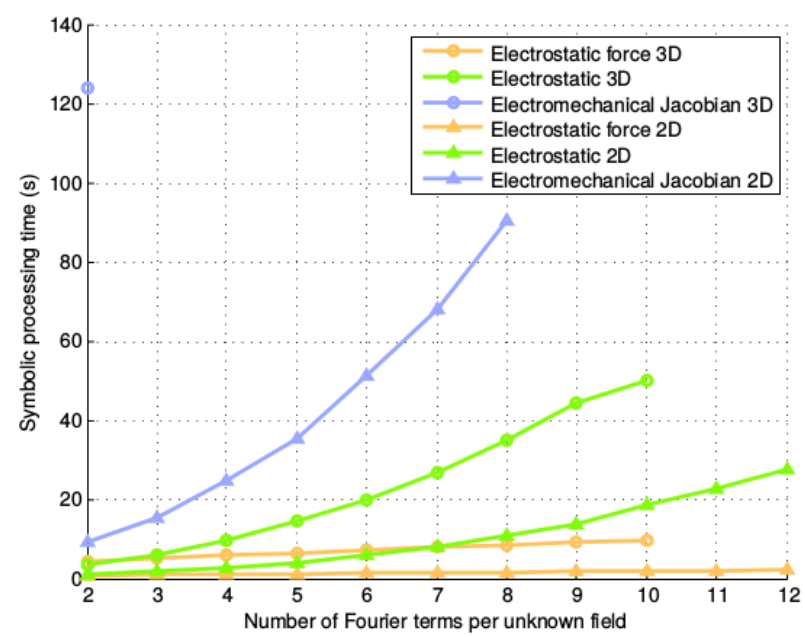

Fig. 8. Time (s) for the symbolic multiharmonic computation versus number of terms in each unknown field Fourier expansion.

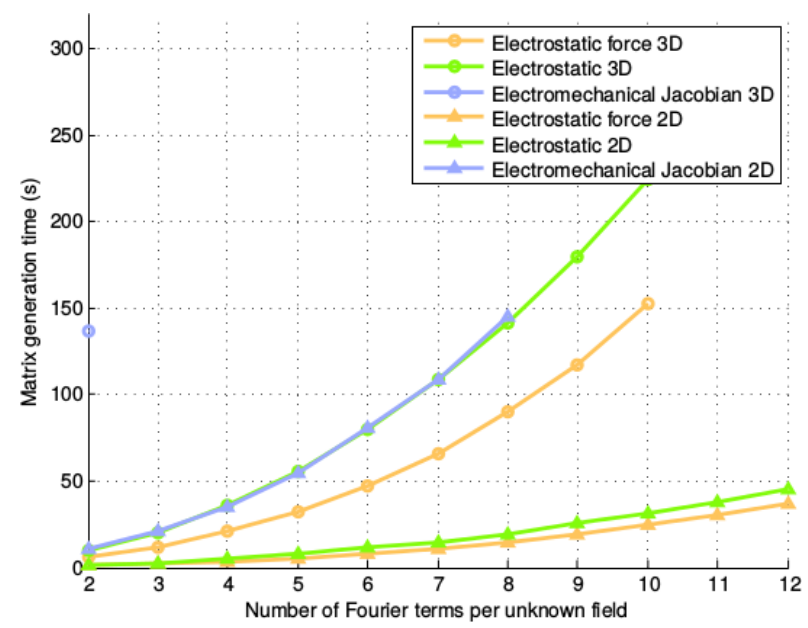

Fig. 9. Matrix generation time (s) versus number of terms in each unknown field Fourier expansion. 


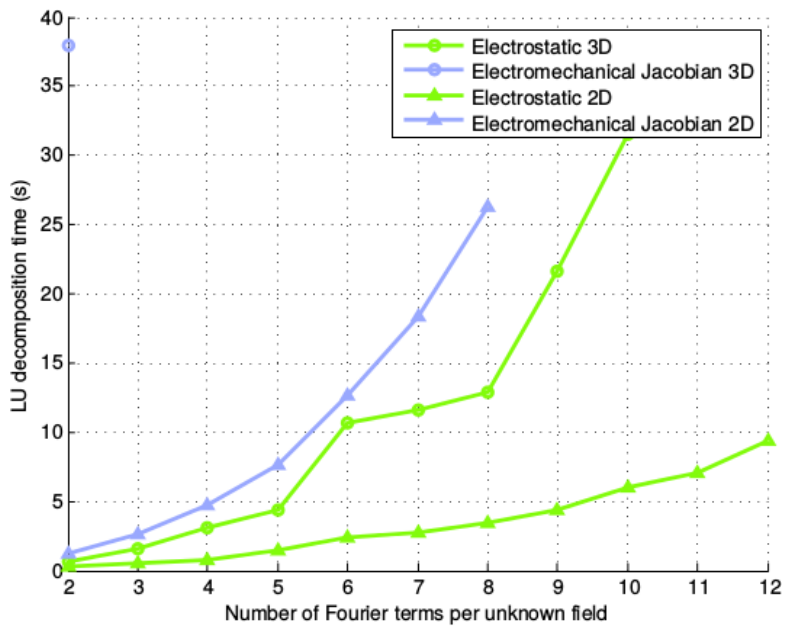

Fig. 10. Time (s) for the LU decomposition versus number of terms in each unknown field Fourier expansion.

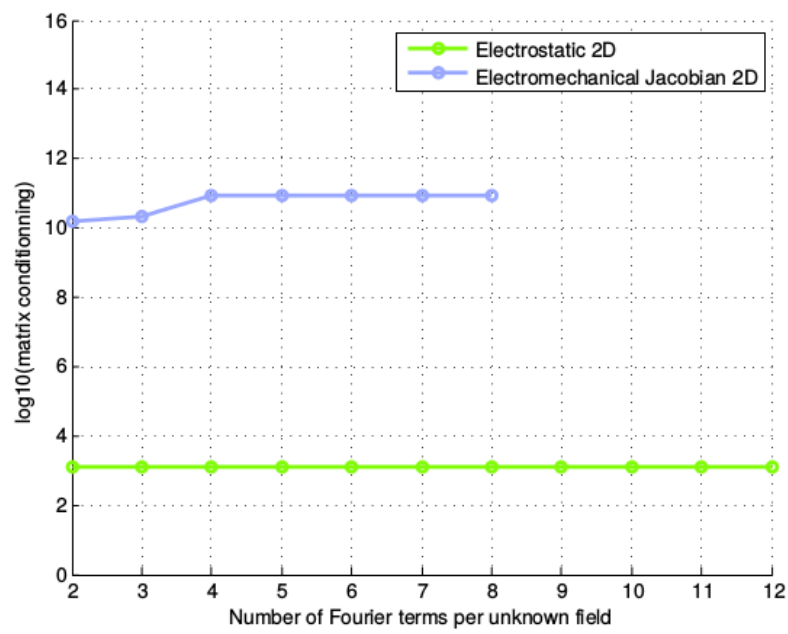

Fig. 11. Condition number versus number of terms in each unknown field Fourier expansion.

the original time-domain solution of the staggered electromechanical formulation obtained using a Newmark scheme. In all cases the very large number of timesteps required to approach steady-state with the timestepping approach makes both multiharmonic resolutions (staggered and monolithic) orders of magnitudes faster. When the driving voltage is low, the staggered multiharmonic scheme is computationally more efficient than the monolithic one. Close to pull-in, i.e. with a stronger nonlinearity, the opposite is true. Accuracy wise, two or three harmonics are often sufficient for the problem at hand with a purely sinusoidal excitation (see Fig. 7). In order to study the computational efficiency in terms of the number of harmonics, we show in Fig. 811 the time to derive the multiharmonic formulation using symbolic computation, the time to generate the multiharmonic matrices, the time to perform a LU decomposition of these matrices and the condition number of the matrices versus the number of
Fourier terms considered in every unknown field truncated expansion. Results are shown for both the 2D and the 3D test cases. With 16 GB RAM on the test computer the 3D Jacobian matrix could be LU decomposed with 2 harmonics per unknown field while the electrostatic formulation could reach about 10 . In $2 \mathrm{D}$ the limit was at about 8 for the Jacobian matrix.

Fig. 8 and 9 show that the symbolic computation step to derive the multiharmonic formulation takes a non negligeable proportion of the total generation time which itself takes up to 5 times longer than the LU decomposition (displayed on Fig. 10). Note however that the symbolic computation time does not depend on the number of elements in the mesh. Finally, Fig. 11 shows the almost independence of the condition number with the number of Fourier coefficients in the unknown fields. This is an important point since the electromechanical formulation tends to have a bad conditioning because of the huge difference between the mechanical and electrical stiffnesses. (The condition number shown is the condition number of the diagonally scaled matrices [16].)

\section{Conclusions}

This paper has detailed a new method to allow for a multiharmonic resolution of finite element formulations that must be computed on a mesh deformed by a (mechanical) displacement. Tests have been performed for a $2 \mathrm{D}$ as well as a $3 \mathrm{D}$ vibrating micromembrane array using both a staggered nonlinear resolution scheme and a monolithic Newton iteration. In both cases these multiharmonic formulations outperform the classical timestepping solution methods. While the number of terms in the formulations grows significantly with the number of Fourier harmonics considered, a completely automatic derivation of these terms makes it fully transparent to the user. Once basic symbolic computation routines are available, the implementation of the technique only relies on classical finite element technology, and can thus be quite easily integrated in existing codes.

\section{Acknowledgment}

Work supported in part by the Belgian Science Policy under grant IAP P7/02.

\section{References}

[1] V. Rochus and C. Geuzaine. A primal/dual approach for the accurate evaluation of the electromechanical coupling in mems. Finite Elements in Analysis and Design, 49(1):19-27, 2012.

[2] N. M. Newmark. A method of computation for structural dynamics. Journal of the Engineering Mechanics Division, 85(3):67-94, 1959.

[3] K-J. Bathe and E. L. Wilson. Numerical methods in finite element analysis. 1976.

[4] F. Bachinger, U. Langer, and J. Schöberl. Numerical analysis of nonlinear multiharmonic eddy current problems. Numerische Mathematik, 100(4):593-616, 2005.

[5] D. M. Copeland and U. Langer. Domain decomposition solvers for nonlinear multiharmonic finite element equations. Journal of Numerical Mathematics, 18(3):157-175, 2010. 
[6] F. Bachinger, U. Langer, and J. Schöberl. Efficient solvers for nonlinear time-periodic eddy current problems. Computing and Visualization in Science, 9(4):197-207, 2006.

[7] F. Bachinger, M. Kaltenbacher, and S. Reitzinger. An efficient solution strategy for the hbfe method. Proceedings of the IGTE, 2:385-389, 2002.

[8] H. De Gersem, H. Vande Sande, and K. Hameyer. Strong coupled multi-harmonic finite element simulation package. COMPELThe international journal for computation and mathematics in electrical and electronic engineering, 20(2):535-546, 2001.

[9] J. Gyselinck, P. Dular, C. Geuzaine, and W. Legros. Harmonicbalance finite-element modeling of electromagnetic devices: a novel approach. Magnetics, IEEE Transactions on, 38(2):521-524, 2002.

[10] G. Paoli, O. Biro, and G. Buchgraber. Complex representation in nonlinear time harmonic eddy current problems. Magnetics, IEEE Transactions on, 34(5):2625-2628, 1998.

[11] S. Yamada and K. Bessho. Harmonic field calculation by the combination of finite element analysis and harmonic balance method. Magnetics, IEEE Transactions on, 24(6):2588-2590, 1988.
[12] A. Halbach, P. Dular, and C. Geuzaine. Comparison of nonlinear domain decomposition schemes for coupled electromechanical problems. IEEE Transactions on Magnetics, In press, 2015.

[13] G. Li and NR. Aluru. A lagrangian approach for electrostatic analysis of deformable conductors. Microelectromechanical Systems, Journal of, 11(3):245-254, 2002.

[14] J. L. Coulomb. A methodology for the determination of global electromechanical quantities from a finite element analysis and its application to the evaluation of magnetic forces, torques and stiffness. IEEE Transactions on Magnetics, 19(6):2514-2519, 1983.

[15] V. Rochus, D. J. Rixen, and J.-C. Golinval. Monolithical modeling of electro-mechanical coupling in micro-structures. International Journal for Numerical Methods in Engineering, 65(4):474-482, 2006.

[16] S. David A. Hannot. Modeling strategies for electro-mechanical Microsystems with uncertainty quantification. TU Delft, Delft University of Technology, 2010. 